\title{
Notes sur quelques pâturages camelins et la cure de sel dans l'Adrar des Iforas et la région de Tombouctou
}

\author{
(Rapport de M. JOUSSELIN)
}

DuRANT une tournée d'information, nous avons pu obtenir un certain nombre de renseignements, concernant les pâturages camelins, ainsi que des indications sur la succession de ces pâturagés. Cette succession définit, pour chaque région, le cycle de nomadisation annuel. Nous ne sommes resté qu'assez pou de temps dans ces régions et nous n'avons pu contrôler tous ces renseignements qui nous furent fournis oralement par des administrateurs, des officiers et des sous-officiers commandant, soit des régions habitées par des nomades soit des unités méharistes.

Les noms des différentes plantes fourragères ne nous furent indiqués qu'en dialecte local. Nous avons essayé d'obtenir le maximum de correspondance arabe et tamacheq, afin de pouvoir nous reporter avec plus de précisions aux références botaniques existantes sur ces régions, et nous permettre de donner une allure plus rationnelle à nos notes. Il ne s'agit donc nullement d'un travail scientifique malgré les appellations latines des végétaux. N'ayant emporté dans cette tournée aucun matériel d'herbier, nous n'avons pu réaliser que de très mauvaises collections qui ne purent guère nous aider pour la détermination botanique. En conséquence, nous ne donnons ces renseignements qu'avec un gros coefficient d'erreur, la précision ne pouvant aller au-delà du genre botanique.

\section{I. - SUCCESSION DES PATURAGES CAMELINS DANS L'ADRAR DES IFORAS}

L'Adrar des Iforas est un massif montagneux, granitique dans son ensemble, les nombreuses vallées contiennent des dépôts alluvionnaires qui constituent le sol des pâturages de la région. Ces pâturages sont très riches comparativement à ceux d'un pays de dunes comme l'Azaouad de Tombouctou.

De mai à mi-juillet : espèces dominantes :

Taguinguillit (Temacheq) = Eidiat (Arabe). Papilionacée : Tephrosia polystachia.

Tagmet ( $T$ ) non déterminé.

Eflich $(\AA)=$ Tekaneit $(T)$. Acanthacée : Blepharis edulis.

De fin juillet à début août (début de la saison des pluies) :
Afazour $(T)=$ Marcoubah $(A)$. Graminée : Panicum turgidum (vert).

Pâturages d'arbres.

D'août à octobre (saison des pluies, pâturages verts d'oueds) :

Taguerouft (T). Rutacée : Tribulus terrestris.

Taguinguillit $(\mathrm{T})=$ Eidiat $(\mathrm{A})$. Papilionacée : Tephrosia polystachia.

Amedressal (T) $=$ Aguertin (A). Ficoïde : Trianthema pentendra.

Imsiken (T). Convolvulacée : Convolvulus fatmensis.

Tekaneit $(T)=$ Eflich $(A)$. Acanthacée: Blepharis edulis.

De fin octobre au début novembre :

Pâturages d'arbres.

Paille de graminées.

De mi-novembre à mi-mars : nomadisation vers le Nord.

Jir-jir $(A)=$ Ahelouat $(T)$. Crucifère : Shouwia arabica.

Pâturages d'arbres.

Paille.

De mi-mars à mai : pâturages plus au nord que les précédents.

Had $(A)=$ Tachara $(\mathrm{I})$. Chénopodiacée : Cornulaca monacantha; pâturage salé associé avec des pâturages d'oueds et d'arbres.

Cure de sel. - A titre dépuratif, les nomades font absorber à leurs animaux, à époques fixes de l'année, du chlorure de sodium et des sels magnésiens. Cette cure se pratique, soit en conduisant le troupeau à certains puits connus et réputés, soit en faisant ingérer des terres salées provenant de régions particulières.

Pour l'Adrar des Iforas, le puit salé est celui d'Asselar. Les indigènes font en général faire deux cures de sel par an : une avant, l'autre après la saison des pluies. Cette cure consiste à faire cinq abreuvoirs à cinq ou six jours d'intervalle.

En dehors de la cure de sel proprement dite, les chameliers ont coutume de donner tous les quinze ou vingt jours une terre salée provenant de la région de Tessalit, à raison d'un plat de campement pour sept dromadaires. 


\section{II. - SUCCESSION DES PATURAGES DANS L'AZAOUAD DE TOMBOUCTOU}

De mai à juillet : saison très sèche.

Cram-cram sec $=$ Initi $(A)=$ Ouazzai $(T)$. Graminée: Cenchrus catharticus: pâturage de dune.

De juillet à septembre : saison des pluies.

Cram-cram vert et sec dans la région du fleuve.

De septembre à novembre : les animaux remontent vers le Nord.

Telebout $(\mathrm{T})=$ Taliguit $(\mathrm{T})$. Cypéracée: Cyperus conglomeratus.

Amasmaz $(A)=$ Isersan $(\mathrm{T})$. Papilionacée : Tephrosia purpurea,

Asebaie $(A)=$ Ana $(T)=$ Titarek (Maure). Asclépiadée : Leptadenia spartum.

De novembre à mars : les animaux remontent toujours vers le Nord.

Tejao $(\mathrm{A})=$ Tachaout $(\mathrm{T})$. Papilionacée: Indigofera semitrijuga.

Eflich $(\AA)=$ Tekaneit $(T)$. Acanthacée $:$ Blepharis edulis

Sbott $(\mathrm{A})=$ Tossouyé (T). Graminée : Aristida pungens.

De mars à mai : pâturages au nord de la région de nomadisation.

Had $(A)=$ Tachara $(T)$. Chénopodiacée: Cornulaca monacantha, pâturage salé.

Cure de sel. - Se fait dans les pâturages salés de had et en distribuant du sel de troisième qualité de Taoudéni.

\section{III. - SUCCESSION DES PATURAgES DANS LA RÉGION DE TAHOUA}

De mai à juillet : saison sèche.

Cram-cram sec, Cenchrus catharticus et paille d'autres graminées.

De juillet à septembre : saison des pluies, pâturages au sud de la région de nomadisation.

Cram-cram vert. Graminée : Cenchrus catharticus.

Taguerouft (T). Rutacée : Tribulus terrestris.

Amedressal (T). Ficoïde : Trianthema pentendra.

Eïdiat $(\mathrm{A})=$ Taguinguillit $(\mathrm{T})$. Papilionacée : Tephrosia polystachia.

Imsiken ( $\mathrm{T}$ ). Convolvulacée : Convolvulus fatmensis.

De septembre à novembre: troupeaux remontant vers le Nord.

Telebout $(\mathrm{T})=$ Taliguit $(\mathrm{T})$. Cypéracée : Cyperus conglomeratus.

Eglech $(A)=$ Timougioust (T). Zygiophyllacée du genre Tribulus.
De novembre à mars : troupeau remontant vers le Nord.

Eglech $(A)=$ Timougloust $(T)$. Zygophyłlacée du genre Tribulus.

Jir-jir $(A)=$ Ahelouat $(T)$. Crucifere : Shouwia arabica.

De mars à mai : animaux au nord de leur zone de nomadisation.

Had $(\mathrm{A})=$ Tachara $(\mathrm{T})$. Chónopodiacée: Cornulaca monocantha.

Cure de sel. - Se fait dans le pâturage salé de had et, paraît-il, dans des puits de la région de Bilma.

\section{IV. - SUCCESSION DES PATURAGES DANS LA RÉGION DE L'AZHOUAD, DE GOUNDAM ET DU NORD FAGUIBINE}

Région de dunes avec des pâturages très maigres : dans le sud de lá zone de nomadisation, pâturages plus riches des bords du fleuve et des fonds de lacs (Faguibine, Gouber, Horo, etc.).

De mai à juillet : saison sèche.

Eflich $(\mathrm{A})=$ Tekaneit $(\mathrm{T})$. Acanthacé : Blepharis edulis.

Akchit $(A)=$ Oussit (T). Crucifère' du genre Farsetia.

Initi $(A)=$ Ouazzaï $(T)=$ Cram-cram sec. Graminée : Cenchrus catharticus.

Atil $(A)=$ Agjar $(T)=$ arbre de la famille des Capparidées, genre Moerua.

Sbib lougrouf $(A)$. Polygalée, genre Polygala.

De mi-juillet au début août : début de la saison des pluies.

Tala $(\Lambda)$ et pâturages d'autres mimosées arbustives.

Tougourit $(\mathrm{T})=$ Askanit $(A)$. Graminée: Latipes senegalensis.

Début août à septembre : saison des pluies.

Sag el mohor $(A)=$ Teloloud $(A)$. Graminée : Aristida stipoldes.

Gasbah $(A)=$ Akhallas $(T)=$ Cranz-cram vert. Graminée : Cenchrus catharticus.

Eflich $(A)=$ Tekaneit $(T)$. Acanthacee : Blepharis edulis.

Amsrar $(A)=$ Ameterar (T). Molluginacée : Gisekia pharnacoïdes.

Tougourit $(\mathrm{T})=$ Askanit $(\mathrm{T})$. Graminé : Latipes senegalensis.

Pâturages d'arbres.

De septembre à octobre : troupeau au voisinage du fleuve.

Mêmes pâturages que les précédents; mais plus secs.

En plus : Amasmaz (A) = Isersan (T). Papilionacée du genre Tephrosia. 
De novembre à avril : les animaux remontent vers le Nord.

Telebout $(\mathrm{T})=$ Taliguit $(\mathrm{T})$. Cypéracée : Cyperus conglomeratus.

Gasbah $(A)=$ Akhallas $(T)=$ Cram-cram vert. Graminee : Cenchrus catharticus.

Aouarach (T). Polygonacée : Calligonum comosum.

Tejao $(\mathrm{A})=$ Tachaout $(\mathrm{T})$. Papilionacée: Indigofera semitrijuga.

Akchit $(A)=$ Oussit $(T)$. Crucifère du genre Farsetia.

Amasmaz $(\AA)=$ Isersan (T). Papilionacée du genre Tephrosia.

Ergueboulé (A).

Pâturages d'arbres.

Had.

Serdoum.

D'avril à mai : les animaux redescendent vers le fleuve, après la cure salée dans les pâturages de had,

Cram-cram sec $=$ Ouazzaï $(\mathrm{T})=$ Initi $(A)$. Graminée : Cenchrus catharticus.

Cure de sel. - Dure quinze jours, se pratique de fin novembre au début de décembre. Pâturage de had, Chénopodiacée, Cornulaca monocantha, associée au Serdoum $(A)=$ Taranmout $(T)$. Graminée : Aristida acutifolia.

Pendant le courant de l'année, distribution de sel de Taoudeni de troisième qualité, tous les vingt jours environ.

\section{v. - SUCCESSION DES PATURAGES DANS LE HODH}

De mars au 15 juillet : pâturages au nord de Nema.

Cram-cram sec $=$ Initi $(A)=$ Ouazzaï $(T)$. Graminée: Cenchrus catharticus.

Amasmaz $(A)=$ Isersan $(T)$. Papilionacée genre Tephrosia.

Sbib lougrouf. Polygalée genre Polygola.

Eflich $(A)=$ Tekaneït $(\mathrm{T})$. Acanthacée: Blepharis edulis.

Pâturages d'arbres.
De mi-juillet à mi-août :

Pâturages d'arbres :

Adres (A). Burséracée: Commiphora africana.

Tamat (A). Mimosée : Acacia seyal, etc.

De mi-août à octobre : animaux remontant vers le Nord.

Eflich $(A)=$ Tekaneït $(T)$. Acanthacée : Blepharis edulis.

Gasbah (A) - Akhallas (T). Graminne: Cenchrus catharticus vert.

Amsrar $(A)=$ Ameterar $(\mathrm{T})$. Molluginacée : Gisekia pharnacoïdes.

Tartaga (A). Papilionacée : Crotalaria arenaria.

D'octobre à mi-novembre : pâturages de la région de Oualata.

Telebout $(\mathrm{T})=$ Taliguit. Cypéracée : Cyperus conglomeratus.

Amsrar (A) $=$ Ameterar (T), Molluginacée : Gisekia pharnacoïdes.

Amasmaz $(\mathrm{A})=$ Isersan $(\mathrm{T})$. Papilionacée genre Tephrosia.

Tleah $(\mathrm{A})=$ Tamat $(\mathrm{T})$. Mimosée : Acacia seyal.

De mi-novembre à mi-mars : pâturages au nord de Oualata.

Telebout $(\mathrm{T})=$ Taliguit $(\mathrm{T})$. Cypéracée: Cyperus conglomeratus.

Had (A). Chénopodiacée : Cornicula monacantha.

Serdoum $(A)=$ Taranmout $(T)$, Graminée : Aristida acutifolia.

Akchit $(\mathrm{A})=$ Oussit $(\mathrm{T})$. Crucifère du genre Farsetia.

Tejao $(\mathrm{A})=$ Tachaout $(\mathrm{T})$. Papilionacáe: Indigofera semitrijuga.

Core de sel. - Se fait de mars à juillet; ne dure que quinze jours, se fait au puits de Taguigal, qui est fortement natroné.

Tous les vingt jours, pendant le courant de l'année, il est distribué aux dromadaires du sel de Tinioulif. L'animal doit s'accoutumer à ces ingestions; la première fois, il n'en absorberait que 50 grammes, puis 100 grammes la fois suivante pour arriver jusqu'à 400 grammes 